\title{
Characterization of complications associated with open and endoscopic craniosynostosis surgery at a single institution
}

\author{
Rowland H. Han, MSE, ${ }^{1}$ Dennis C. Nguyen, MD, ${ }^{2}$ Brent S. Bruck, BS, ${ }^{1}$ Gary B. Skolnick, BS, ${ }^{2}$ \\ Chester K. Yarbrough, MD, MPHS, ${ }^{1}$ Sybill D. Naidoo, PhD, RN, CPNP, ${ }^{2}$ Kamlesh B. Patel, MD, ${ }^{2}$ \\ Alex A. Kane, MD, ${ }^{3}$ Albert S. Woo, MD, ${ }^{2}$ and Matthew D. Smyth, MD ${ }^{1}$ \\ 1Department of Neurological Surgery and 'Department of Surgery, Division of Plastic and Reconstructive Surgery, Cleft \\ Palate-Craniofacial Institute, Washington University School of Medicine in St. Louis, Missouri; and 'Department of Plastic \\ Surgery, University of Texas Southwestern Medical Center, Dallas, Texas
}

\begin{abstract}
OBJECTIVE The authors present a retrospective cohort study examining complications in patients undergoing surgery for craniosynostosis using both minimally invasive endoscopic and open approaches.

METHODS Over the past 10 years, 295 nonsyndromic patients (140 undergoing endoscopic procedures and 155 undergoing open procedures) and 33 syndromic patients (endoscopic procedures in 10 and open procedures in 23) met the authors' criteria. Variables analyzed included age at surgery, presence of a preexisting CSF shunt, skin incision method, estimated blood loss, transfusions of packed red blood cells, use of intravenous steroids or tranexamic acid, intraoperative durotomies, procedure length, and length of hospital stay. Complications were classified as either surgically or medically related.
\end{abstract}

RESULTS In the nonsyndromic endoscopic group, the authors experienced $3(2.1 \%)$ surgical and $5(3.6 \%)$ medical complications. In the nonsyndromic open group, there were $2(1.3 \%)$ surgical and $7(4.5 \%)$ medical complications. Intraoperative durotomies occurred in $5(3.6 \%)$ endoscopic and $12(7.8 \%)$ open cases, were repaired primarily, and did not result in reoperations for CSF leakage. Similar complication rates were seen in syndromic cases. There was no death or permanent morbidity. Additionally, endoscopic procedures were associated with significantly decreased estimated blood loss, transfusions, procedure length, and length of hospital stay compared with open procedures.

CONCLUSIONS Rates of intraoperative durotomies and surgical and medical complications were comparable between endoscopic and open techniques. This is the largest direct comparison to date between endoscopic and open interventions for synostosis, and the results are in agreement with previous series that endoscopic surgery confers distinct advantages over open surgery in appropriate patient populations.

http://thejns.org/doi/abs/10.3171/2015.7.PEDS15187

KEY WORDS craniosynostosis; craniofacial; surgical complications; endoscope; minimally invasive

$\mathrm{O}$ VER the past decade, the use of minimal incision craniectomy for craniosynostosis has increased as an alternative to full-exposure craniectomy or calvarial vault reconstruction. ${ }^{2,30}$ Differences in utilization and opinions regarding outcomes of these techniques differ among surgeons specializing in the care of these conditions. ${ }^{6,23}$ Outcome variables debated include magnitude and durability of head shape improvement, cost, neurodevelopmental trajectory, burden of care to the patient, and intra- and postoperative complication rates. ${ }^{4,31}$ The purpose of this work was to ascertain the incidence of intra- and postoperative complications in a single center. Although a number of studies have reported on various metrics of success in endoscopic ${ }^{9,12,14}$ or open ${ }^{8,27}$ craniosynostosis surgeries, few have directly compared results of the two on a large cohort of patients. ${ }^{5,16}$ We present a ret-

ABBREVIATIONS EBL = estimated blood loss; EBV = estimated blood volume; PRBC = packed red blood cells; $T$ TXA = tranexamic acid.

SUBMITTED March 30, 2015. ACCEPTED July 17, 2015.

INCLUDE WHEN CITING Published online November 20, 2015; DOI: 10.3171/2015.7.PEDS15187. 
rospective study examining complications after surgery in nonsyndromic and syndromic cases of craniosynostosis.

\section{Methods \\ Study Design}

This retrospective cohort study at St. Louis Children's Hospital determined rates and causes of complications in patients undergoing endoscopic and open craniosynostosis surgery between 2003 and 2013. All patients undergoing these operations during the study period were included in the analysis. The study compared complications related to age at surgery, affected sutures, presence of preexisting CSF shunt, skin incision method, estimated blood loss (EBL), intraoperative and postoperative transfusions of packed red blood cells (PRBC), use of intravenous steroids or tranexamic acid (TXA), procedure length, length of hospital stay, and readmissions within 30 days. The Washington University Human Research Protection Office approved this study.

\section{Study Patients}

Eligible patients included those with sagittal, metopic, unicoronal, lambdoid, bicoronal, squamosal, or multisuture craniosynostosis diagnosed by a craniofacial team that included 1 pediatric neurosurgeon (M.D.S.) and 3 plastic surgeons (K.B.P., A.A.K., and A.S.W.). Craniosynostosis was diagnosed through a combination of physical examination, skull radiographs, and 3D head CT scans. Our center routinely obtains a preoperative, low-dose 3D head CT scan in patients with suspected craniosynostosis if not already performed prior to referral. All patients fulfilling these criteria were included regardless of clinical follow-up length. We classified events as surgical complications (intraoperative, excluding durotomies), intraoperative durotomies, or medical complications (postoperative). Separate analyses were conducted for syndromic and nonsyndromic craniosynostosis cases.

\section{Operative Technique}

Both endoscopic and open procedures were offered to all patients presenting earlier than 6 months of age. We considered comorbidities, syndromes, and anticipated difficulties with postoperative helmet molding before making a final determination to use the endoscopic approach. Patients who presented after 6 months of age were offered an open operation only. Because it is the most common type of nonsyndromic single-suture craniosynostosis, we describe the open and endoscopic procedures for premature fusion of the sagittal suture below.

\section{Cranial Vault Remodeling for Sagittal Craniosynostosis}

We use a bicoronal zigzag incision posterior to the coronal suture and behind the insertion of the temporalis muscle. A subperiosteal plane is developed to expose the cranial vault. The scalp and periosteum are retracted to expose the frontal and parietal bones. Next, we perform bilateral parietal (with or without frontal) craniotomies and use Tessier bone benders and osteotomies to reshape the cranial vault. After contouring, we secure the bones with an absorbable plating system. We place a subgaleal drain in most open cases. The technique varies slightly depending on the craniofacial plastic surgeon involved. Additional details have been previously published. ${ }^{28}$

\section{Endoscope-Assisted Craniectomy for Sagittal Craniosynostosis}

For endoscopic suturectomy, we use a transverse incision just posterior to the anterior fontanelle and another transverse incision just anterior to the lambda. We then place bur holes at each incision, strip the dura from the inner table of the skull, and use curettes and rongeurs to remove bone across the midline. We then strip the dura assisted by the endoscope to allow for our craniectomies and any other planned osteotomies. After the planned bone is removed, we use electrocautery and hemostatic agents to obtain hemostasis. From 2006 to 2011 we performed wide vertex craniectomies with parietal wedge osteotomies as described in detail by Jimenez and Barone, ${ }^{15,28}$ and since 2011 have switched to narrow strip craniectomies as detailed by Proctor and colleagues. ${ }^{25}$

\section{Hospital Course and Follow-Up}

Patients who underwent open procedures were admitted to the pediatric ICU overnight with postoperative hemoglobin and hematocrit monitoring to determine whether transfusion was required. Typically, blood products were given in the operating room, but postoperative transfusions were performed when a patient was symptomatic or had a hematocrit lower than $21 \%-24 \%$, depending on subgaleal drain output and overall status. Patients received antibiotic prophylaxis as long as the subgaleal drain was in place. These patients were evaluated 3 weeks after discharge, and the majority returned for a 1-year follow-up evaluation.

Patients undergoing endoscopic operations were admitted to the neurosurgical ward and also underwent postoperative hemoglobin and hematocrit monitoring. In general, these patients were observed and discharged to home the next day. The threshold for transfusion was a hematocrit of less than $18 \%$. The patients were fitted for cranial molding helmets in the 1st week after surgery and received continuing helmet therapy under the supervision of orthotists until 12 months of age. During this time, they typically outgrew 1 helmet and used 2 or 3 total. ${ }^{22,31}$ Patients who underwent endoscopic operations were evaluated every 2-3 months by our team to ensure adequate cranial remodeling.

During the latter part of the series, dexamethasone was used for scalp and facial edema, and TXA was used to minimize intraoperative blood loss in some cases, but not in a standardized fashion.

\section{Data Collection and Statistical Analysis}

For each patient, we reviewed all available clinical data including intraoperative surgery and anesthesia notes, ICU progress reports, laboratory results, helmet-fitting appointments, clinical evaluations, and reoperations. Estimated blood loss was obtained from anesthesia records, and a weight-based calculation was made for estimated 
blood volume (EBV). For infants younger than 2 years old, $\mathrm{EBV}=$ (weight in $\mathrm{kg}) \times(80 \mathrm{ml} / \mathrm{kg})$. The percentage of EBV lost during the surgical procedure $(\mathrm{EBL} / \mathrm{EBV}$ ratio $=$ $\mathrm{EBL} / \mathrm{EBV} \times 100 \%$ ) was then calculated. ${ }^{31}$

All events were classified as surgical complications, intraoperative durotomies, or medical complications (Table 1). Data for continuous variables were presented as the mean \pm 1 SD. Statistical computations were performed using SPSS (version 22, IBM Corp.) with Student t-test for continuous variables or Pearson's chi-square test for categorical data. Dunn-Šidák correction for multiple comparisons was applied based on an original $\alpha=0.05$ to determine statistical significance. Significance levels are listed separately on each relevant table.

\section{Results \\ Patient Population}

In the 10-year span, 295 nonsyndromic craniosynostosis patients (140 endoscopic and 155 open surgeries) and 33 syndromic patients (10 endoscopic and 23 open surgeries) underwent surgical correction performed by our craniofacial team. For each of these patients, only initial operations to correct synostosis were included in our analysis. Of the nonsyndromic cases, patients receiving endoscopic surgery had a mean age at surgery of $3.4 \pm 1.2$ months (mean \pm SD, Fig. 1) and were followed postoperatively for a duration of $25.2 \pm 19.3$ months (range 0.4-88.1 months), while those undergoing open procedures had a mean age at surgery of $15.5 \pm 16.5$ months (Fig. 1) and were followed postoperatively for $37.4 \pm 30.2$ months (range 0.1-125.4 months). None of the nonsyndromic patients who underwent endoscopic procedures had preexisting shunts for comorbid hydrocephalus, while $4(2.6 \%)$ of the patients who underwent open procedures had preexisting CSF shunts (Table 2). In fact, the presence of shunt-treated hydrocephalus precluded us from offering an endoscopic approach due to concerns that head shape may not normalize while the draining force of the shunt counteracts brain expansion required for remodeling in the molding helmet.

Syndromic patients included those with chromosomal deletions and Goldenhar, Crouzon, Charcot-Marie-Tooth, Saethre-Chotzen, DiGeorge, Down, VATER (vertebral anomalies, anal atresia, tracheoesophageal fistula and/or esophageal atresia, renal and radial anomalies and limb defects), and CDAGS (craniosynostosis, anal anomalies, porokeratosis) syndromes. Syndromic patients undergoing endoscopic procedures had a mean age at surgery of $3.8 \pm$ 1.3 months (Fig. 1) and were followed up postoperatively for 36.8 \pm 26.2 months (range 2.3-74.9 months). Syndromic patients undergoing open procedures had a mean age at surgery of $41.4 \pm 58.3$ months (Fig. 1) and were followed up postoperatively for $36.9 \pm 28.5$ months (range 0.1-113.6 months). Of our syndromic patients, 1 of the 10 endoscopic cases and 4 of the 23 open cases had preexisting shunts (Table 3).

\section{Suture Locations}

Early in the development of our endoscopic program we offered minimally invasive procedures more often to patients with sagittal synostosis and later began to also offer them for coronal and metopic fusions as we gained experience and confidence in the technique. Therefore, our series included a higher rate of sagittal synostosis (94 patients [67.1\%] in the endoscopic group and 76 [49.0\%] in the open group) and a lower rate of unicoronal synostosis (10 patients [7.1\%] in the endoscopic group and 28 [18.1\%] in the open group). Rates of metopic, lambdoid, bicoronal, and multiple (2-suture) synostosis were nevertheless comparable between the two groups. We used open procedures for $3(1.9 \%)$ patients with 3 -suture synostosis and $1(0.6 \%)$ patient with 4-suture synostosis. None of these more severe nonsyndromic multisuture cases underwent endoscopic treatment (Table 4). Of our 10 syndromic endoscopic cases, 4 had single-suture, 5 had 2-suture, and 1 had $\geq 3$-suture synostosis. Within our 23 syndromic patients in the open group, 11 had single-suture, 6 had 2-suture, and 5 had $\geq 3$-suture synostosis (Table 3 ).

\section{Skin Incisions}

We examined methods used to make the initial skin incision in all patients with available data. Incisions were either performed sharply (scalpel) or with Colorado needle tip electrocautery. Some patients had partial incisions with both methods and we included them in the scalpel and unipolar electrocautery groups. Among nonsyndromic patients, $52.9 \%$ of endoscopic cases had skin incisions with scalpel, compared with only $5.7 \%$ with electrocautery. The open nonsyndromic cases were more evenly distributed, with $36.8 \%$ having skin incisions with scalpel and $41.3 \%$ with electrocautery (Table 2). Of the syndromic patients with available data, 5 endoscopic cases used a scalpel and 3 used electrocautery. Likewise, 5 syndromic open cases used a scalpel and 12 used electrocautery (Table 3).

\section{Surgical Procedures}

All subjects' clinical data were reviewed for EBL, as

TABLE 1. Classification of events

\begin{tabular}{ll}
\hline \multicolumn{1}{c}{ Group } & Events \\
\hline Surgical complications & $\begin{array}{c}\text { Scalp abscess, oxygen desaturation, intraop reintubation, hemodynamic instability, CSF leak, } \\
\text { cranial defect, dural dehiscence }\end{array}$ \\
\hline Intraoperative durotomies & Intraop durotomies \\
\hline Medical complications & $\begin{array}{c}\text { Stridor, upper airway obstruction, tachycardia, bradycardia, coagulopathy, hypertension, erythema, } \\
\text { continued facial edema, seizure, fever, upper respiratory infection, pneumonia, urinary tract } \\
\text { infection, allergic reaction }\end{array}$ \\
\hline
\end{tabular}



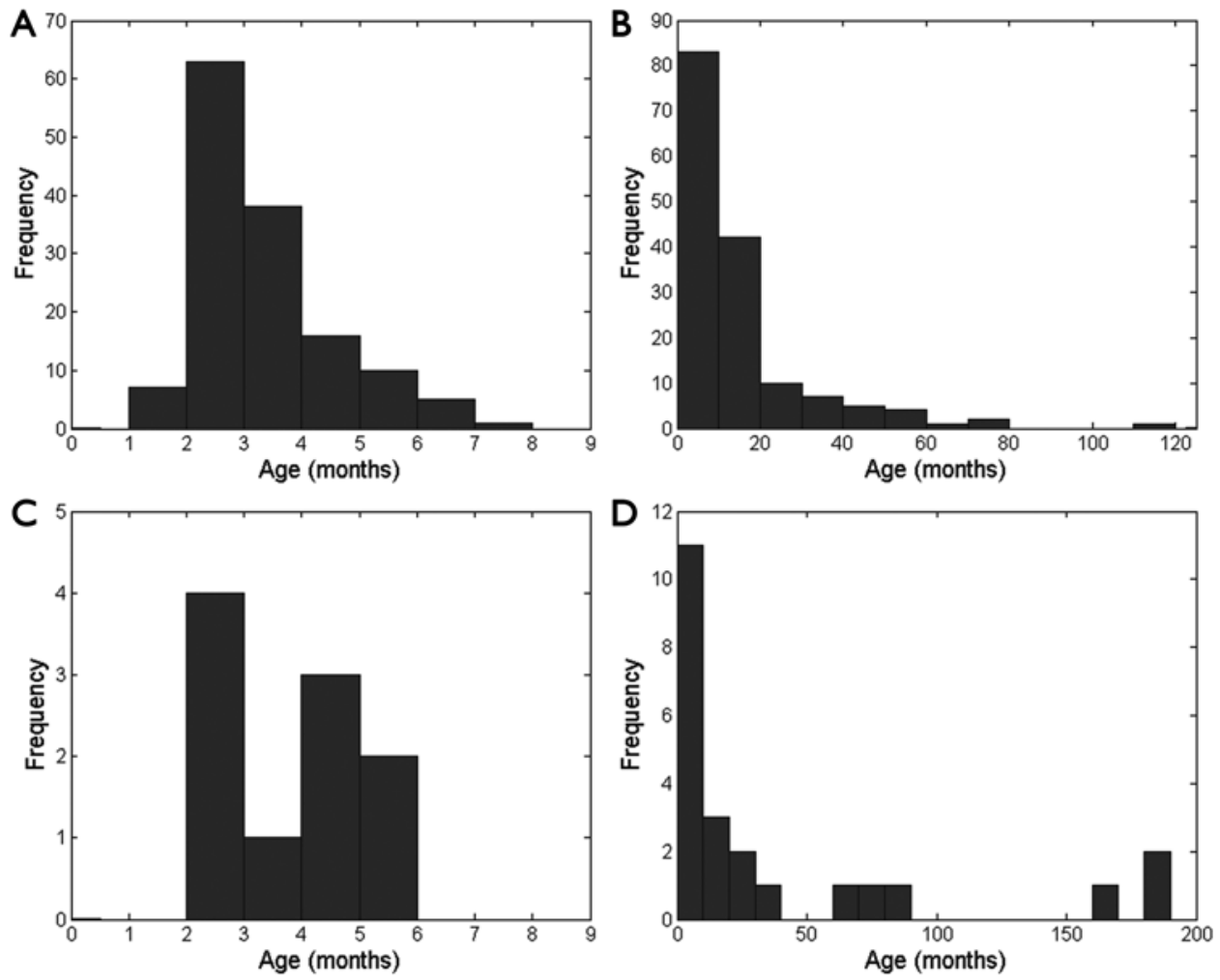

FIG. 1. Bar graphs showing the frequencies of patient ages comparing nonsyndromic endoscopic (A), nonsyndromic open (B), syndromic endoscopic (C), and syndromic open (D) procedures. The distributions reveal that within both nonsyndromic and syndromic groups, patients undergoing endoscopic procedures were significantly younger than those undergoing open operations (nonsyndromic $p<0.001$, syndromic $p=0.005$, Student t-test).

well as PRBC, intravenous dexamethasone, and TXA usage during the intraoperative period. Additionally, we analyzed the average lengths of the various procedures and the length of postoperative hospital stay. It should be noted that within the endoscopic nonsyndromic group, 1 patient was excluded from the length of stay calculation because he was an extreme outlier. This patient's case was complex with concomitant hydrocephalus and a tracheostomy, and he had undergone cardiac surgery for a congenital heart defect. His operation was performed as an inpatient rather than as an elective outpatient. He was in the ICU for 43 days and stayed in the hospital for a total of 61 days after his synostosis operation.

Among the remaining nonsyndromic patients in the endoscopic group, we observed an average EBL of 36.1 $\pm 26.9 \mathrm{ml}$, procedure length of $71.3 \pm 24.8$ minutes, and postoperative length of stay of $1.1 \pm 0.3$ days. In this group, 7 (5.0\%) received intraoperative transfusions of PRBC, 7 (5.0\%) received postoperative transfusions, $9(6.6 \%)$ received steroids, and 8 (5.8\%) received TXA. The nonsyndromic patients in the open surgery group had an average of EBL of $293.2 \pm 180.2 \mathrm{ml}$, procedure length of $168.5 \pm$ 126.3 minutes, and length of stay of $3.8 \pm 1.3$ days. Furthermore, 147 (96.1\%) of patients undergoing open surgery received PRBC intraoperatively, 56 (39.4\%) received PRBC postoperatively, 30 (27.0\%) received steroids, and $22(20.2 \%)$ received TXA (Table 2 ).

Focusing on our 10 syndromic patients in the endo- scopic group, we found an average EBL of $42.8 \pm 29.6$ $\mathrm{ml}$, procedure length of $129.7 \pm 99.7$ minutes, and length of stay of $1.4 \pm 0.7$ days. Steroids were given to 2 patients and TXA to 1 . Finally, in our 23 syndromic patients in the open group, we observed average EBL of $488.0 \pm 421.2$ $\mathrm{ml}$, procedure length of $325.6 \pm 130.5$ minutes, and length of stay of $3.6 \pm 1.3$ days. In this group, 5 patients received steroids and 2 received TXA (Table 3 ).

We were interested in determining whether preoperative steroid administration led to shorter hospital stays among our various patient cohorts, but found no statistically significant differences in length of stay in any of our groups (Table 5).

\section{Complications}

We classified events as surgical complications, intraoperative durotomies, or medical complications (Table 1). Complications directly related to the operation itself or occurring during the intraoperative period were considered surgical, and those occurring during postoperative management of the patients were classified as medical. All intraoperative durotomies were repaired primarily through the existing skin incisions and did not result in reoperations for CSF leakage. No mortality or permanent morbidity occurred in any of these cases.

In the nonsyndromic endoscopic group, we experienced $3(2.1 \%)$ surgical complications, 5 (3.6\%) intraoperative durotomies, and $5(3.6 \%)$ medical complications. 
TABLE 2. Summary statistics (nonsyndromic patients)*

\begin{tabular}{|c|c|c|c|c|c|}
\hline Variable & Endoscopic & No. of Patients & Open & No. of Patients & $\mathrm{p}$ Value \\
\hline Mean age at surgery in $\operatorname{mos}^{*} \dagger$ & $3.4 \pm 1.2$ & 140 & $15.5 \pm 16.5$ & 155 & $<0.001$ \\
\hline Preplaced shunt & 0 & 139 & $4(2.6)$ & 155 & 0.125 \\
\hline Scalpel & $74(52.9)$ & 140 & $57(36.8)$ & 155 & 0.006 \\
\hline Bovie & $8(5.7)$ & 140 & $64(41.3)$ & 155 & $<0.001$ \\
\hline Mean EBL in $\mathrm{ml}^{*}$ & $36.1 \pm 26.9$ & 140 & $293.2 \pm 180.2$ & 151 & $<0.001$ \\
\hline Mean EBV in $\mathrm{ml}^{*}$ & $488.5 \pm 81.1$ & 140 & $809.1 \pm 333.1$ & 150 & $<0.001$ \\
\hline Mean EBL/EBV ratio, \%* & $7.5 \pm 5.3$ & 140 & $37.8 \pm 21.4$ & 146 & $<0.001$ \\
\hline \multicolumn{6}{|l|}{ PRBC } \\
\hline Intraop transfusions & $7(5.0)$ & 139 & $147(96.1)$ & 153 & $<0.001$ \\
\hline Postop transfusions & $7(5.0)$ & 140 & $56(39.4)$ & 142 & $<0.001$ \\
\hline Intravenous steroids & $9(6.6)$ & 137 & $30(27.0)$ & 111 & $<0.001$ \\
\hline TXA & $8(5.8)$ & 137 & $22(20.2)$ & 109 & $<0.001$ \\
\hline Mean procedure length in mins* & $71.3 \pm 24.8$ & 140 & $168.5 \pm 126.3$ & 155 & $<0.001$ \\
\hline Mean length of stay in days* & $1.1 \pm 0.3$ & 139 & $3.8 \pm 1.3$ & 151 & $<0.001$ \\
\hline Surgical complications & $3(2.1)$ & 140 & $2(1.3)$ & 155 & 0.671 \\
\hline Intraop durotomies & $5(3.6)$ & 140 & $12(7.8)$ & 154 & 0.140 \\
\hline Medical complications & $5(3.6)$ & 140 & $7(4.5)$ & 154 & 0.773 \\
\hline Readmit $<30$ days & $2(1.4)$ & 140 & $2(1.3)$ & 154 & 0.921 \\
\hline Mean follow-up length in mos & $25.2 \pm 19.3$ (range $0.4-88.1)$ & 140 & $37.4 \pm 30.2$ (range $0.1-125.4$ ) & 155 & $<0.001$ \\
\hline
\end{tabular}

* Level of significance: 0.00285 . Values are presented as the number of patients (\%) unless stated otherwise. Mean values are expressed as means \pm 1 SD. Values in boldface are statistically significant.

$\dagger$ See age distributions in Fig. 1.

Readmissions within 30 days of their operations for any reason related to the procedures occurred in $2(1.4 \%)$ of the patients in this cohort. In the nonsyndromic open group, there were $2(1.3 \%)$ surgical complications, $12(7.8 \%)$ intraoperative durotomies, and 7 (4.5\%) medical complications.
Readmissions within 30 days were recorded in 2 (1.3\%) of nonsyndromic open patients (Table 2). In the syndromic endoscopic group we found 1 intraoperative durotomy, no surgical complications, and no medical complications. Syndromic open cases included 2 surgical complications,

TABLE 3. Summary statistics (syndromic)*

\begin{tabular}{lccr}
\hline \multicolumn{1}{c}{ Variable } & Endoscopic $(\mathrm{n}=10)$ & Open $(\mathrm{n}=23)$ & $\mathrm{p}$ Value \\
\hline Mean age at surgery in most & $3.8 \pm 1.3$ & $41.4 \pm 58.3$ & 0.005 \\
\hline Preplaced shunt & 1 & 4 & 0.521 \\
\hline No. of sutures & Single $(4) ;$ double $(5) ; \geq 3(1)$ & Single (11); double $(6) ; \geq 3(5)$ & 0.626 \\
\hline Scalpel/Bovie & $5: 3$ & $5: 12$ & 0.444 \\
\hline Mean EBL in $\mathrm{ml}$ & $42.8 \pm 29.6$ & $488.0 \pm 421.2$ & $<0.001$ \\
\hline Mean EBV in ml & $461.3 \pm 65.3$ & $1226.3 \pm 1139.3$ & 0.005 \\
\hline Mean EBL/EBV ratio, $\%$ & $8.2 \pm 6.1$ & $44.2 \pm 23.3$ & $<0.001$ \\
\hline Intravenous steroids & 2 & 5 & 0.694 \\
\hline TXA & 1 & 2 & 0.582 \\
\hline Mean procedure length in mins & $129.7 \pm 99.7$ & $325.6 \pm 130.5$ & 0.001 \\
\hline Mean length of stay in days & $1.4 \pm 0.7$ & $3.6 \pm 1.3$ & $<0.001$ \\
\hline Surgical complications & 0 & 2 & 0.510 \\
\hline Intraop durotomies & 1 & 7 & 0.256 \\
\hline Medical complications & 0 & 1 & 0.719 \\
\hline Mean follow-up length in mos & $36.8 \pm 26.2$ (range 2.3-74.9) & $36.9 \pm 28.5$ (range 0.1-113.6) & 0.760 \\
\hline
\end{tabular}

* Syndromes: chromosomal deletions, Goldenhar, Crouzon, Charcot-Marie-Tooth, Saethre-Chotzen, DiGeorge, Down, VATER, CDAGS. Level of significance: 0.00341 . Values are presented as the number of patients (\%) unless stated otherwise. Mean values are expressed as the mean \pm 1 SD. Values in boldface are statistically significant.

$\dagger$ See age distributions in Fig. 1. 
TABLE 4. Suture locations (nonsyndromic)

\begin{tabular}{lccc}
\hline \multirow{2}{*}{ Location } & \multicolumn{3}{c}{ No. of Patients (\%) } \\
\cline { 2 - 4 } & $\begin{array}{c}\text { Endoscopic } \\
(\mathrm{n}=140)\end{array}$ & $\begin{array}{c}\text { Open } \\
(\mathrm{n}=155)\end{array}$ & $\begin{array}{c}\text { Combined } \\
(\mathrm{n}=295)\end{array}$ \\
\hline Sagittal & $94(67.1)$ & $76(49.0)$ & $170(57.6)$ \\
\hline Metopic & $24(17.1)$ & $31(20.0)$ & $55(18.6)$ \\
\hline Unicoronal & $10(7.1)$ & $28(18.1)$ & $38(12.9)$ \\
\hline Lambdoid & $6(4.3)$ & $7(4.5)$ & $13(4.4)$ \\
\hline Bicoronal & $2(1.4)$ & $1(0.6)$ & $3(1.0)$ \\
\hline Multisuture $(2)^{*}$ & $7(5.0)$ & $8(5.2)$ & $15(5.1)$ \\
\hline Multisuture $(3)^{*}$ & 0 & $3(1.9)$ & $3(1.0)$ \\
\hline Multisuture $(4)^{*}$ & 0 & $1(0.6)$ & $1(0.3)$ \\
\hline
\end{tabular}

* Numbers in parentheses indicate number of fused sutures.

7 intraoperative durotomies, and 1 medical complication (Table 3).

\section{Reoperations}

In addition to complications, we also characterized all reoperations that were related to the patients' craniosynostosis surgeries. We did not include operations related to other aspects of their management or follow-up procedures in planned 2-stage operations. Among nonsyndromic patients in the endoscopic group, there were 2 reoperations for cranial defects and 1 for suboptimal aesthetics. In the nonsyndromic open group, we had 1 reoperation for a chronically open wound, 2 for hematomas, 2 for removal of implants (wires, bone morphogenetic protein, or distractors), 2 for cranial defects, and 3 for suboptimal aesthetics. With our syndromic patients in the endoscopic group, we had 3 reoperations for suboptimal aesthetics and 1 for a CSF leak related to a one-time trial of a distractor system. Finally, in our syndromic open cohort, we had 1 reoperation for implant removal, 1 for cranial defects, 6 for suboptimal aesthetics, and 2 for wound infections or abscesses.

\section{Subgroup Analysis for Age Younger Than 150 Days}

We performed a subgroup analysis of all previously described data for patients younger than 150 days old at time of surgery, comparing results of endoscopic and open operations for nonsyndromic cases (Table 6). Although we were not able to fully control for age due to our limited number of subgroup patients undergoing open procedures, the outcomes of this analysis are generally consistent with previous results.

\section{Discussion}

Craniosynostosis refers to premature fusion of one or more cranial vault sutures. ${ }^{24}$ It occurs in as many as 1 in 1700 live births, and can be characterized as single- or multisuture, as well as syndromic or nonsyndromic. ${ }^{10}$ In our study, we found that the most common location of nonsyndromic single-suture synostosis is sagittal $(57.6 \%$, Table 4, combined data), followed in decreasing incidence by metopic (18.6\%), unicoronal (12.9\%), and unilambdoid (4.4\%). Multisuture cases including bicoronal represented $7.5 \%$ of the total. These results are generally in agreement with those from previously published large craniosynostosis series. ${ }^{11,19,29}$

To prevent stigma secondary to altered skull shape, as well as sequelae such as restricted brain growth and raised intracranial pressure resulting in neurological, cognitive, or visual impairment, patients with craniosynostosis should be carefully monitored or treated surgically.,17,18 Surgical management of craniosynostosis has shifted from craniectomy to total cranial vault reconstruction, and more recently back to craniectomy due to the popularization of minimally invasive endoscopic approaches by Barone and Jimenez. ${ }^{1,2,30}$ Benefits of the endoscopic approach over more traditional open techniques include comparable safety and efficacy while allowing shorter operative times, reduced costs due to decreased hospital stays, and fewer blood transfusions. ${ }^{4,21,31}$ However, minimally invasive treatment is only possible in cases in which early diagnosis and compliance with postoperative helmet therapy allow proper manipulation of the thin bones of young infants. ${ }^{4}$ Due to these constraints, surgeons vary widely in their opinions regarding the proper timing and indications for choosing an endoscopic versus open approach, or whether to offer an endoscopic approach at all., 623

Patients at our center are generally offered the endoscopic technique if they are younger than 6 months at time of surgery, ${ }^{15}$ but other factors including comorbidities, ${ }^{3}$ syndromes, ${ }^{20}$ and anticipated difficulties with postoperative helmet molding ${ }^{6,32}$ must be considered as well. While some centers perform minimally invasive craniosynostosis surgery in children 6-9 months of age, our practice has been to limit the use of these endoscopic procedures to children younger than 6 months. ${ }^{26}$

Jimenez and colleagues previously summarized the

TABLE 5. Effect of preoperative steroid administration on length of stay

\begin{tabular}{|c|c|c|c|c|c|}
\hline Group & Mean LOS w/o Steroids (days)* & No. of Patients & Mean LOS w/ Steroids (days) ${ }^{*}$ & No. of Patients & $p$ Value \\
\hline Endoscopic nonsyndromic & $1.1 \pm 0.3$ & 127 & $1.0 \pm 0.0$ & 9 & 0.431 \\
\hline Open nonsyndromic & $3.8 \pm 1.3$ & 79 & $3.7 \pm 1.8$ & 29 & 0.739 \\
\hline Endoscopic syndromic & $1.2 \pm 0.4$ & 6 & $2.0 \pm 1.4$ & 2 & NA \\
\hline Open syndromic & $3.1 \pm 1.0$ & 11 & $4.6 \pm 1.8$ & 5 & NA \\
\hline
\end{tabular}

LOS = length of stay; $N A=$ not applicable.

* Level of significance: 0.0253; $p$ values for the syndromic groups are not given as these results would be underpowered. Mean values are expressed as the means \pm $1 \mathrm{SD}$. 
TABLE 6. Summary statistics (nonsyndromic) for patients younger than 150 days at time of surgery

\begin{tabular}{lcccrr}
\hline \multicolumn{1}{c}{ Variable } & Endoscopic & No. of Patients & Open & No. of Patients & $p$ Value \\
\hline Mean age at surgery in days & $92.6 \pm 24.7$ & 124 & $132.4 \pm 12.1$ & 15 & $<0.001$ \\
\hline Preplaced shunt & 0 & 123 & 0 & 15 & 1.000 \\
\hline Scalpel & $64(51.6)$ & 124 & $1(6.7)$ & 15 & 0.001 \\
\hline Bovie & $7(5.6)$ & 124 & $9(60.0)$ & 15 & $<0.001$ \\
\hline Mean EBL in ml & $37.5 \pm 27.6$ & 124 & $245.7 \pm 133.8$ & 14 & $<0.001$ \\
\hline PRBC & & & & 15 & $<0.001$ \\
\hline Intraop transfusions & $7(5.7)$ & 123 & $15(100.0)$ & 14 & $<0.001$ \\
\hline Postop transfusions & $7(5.6)$ & 124 & $8(57.1)$ & 10 & 0.522 \\
\hline Intravenous steroids & $8(6.6)$ & 121 & $1(10.0)$ & 10 & 0.434 \\
\hline TXA & $7(5.8)$ & 121 & $117.5 \pm 96.2$ & 15 & 0.081 \\
\hline Mean procedure length in mins & $71.0 \pm 25.3$ & 124 & $4.1 \pm 1.8$ & 15 & $<0.001$ \\
\hline Mean LOS in days & $1.1 \pm 0.3$ & 123 & $1(6.7)$ & 15 & 0.292 \\
\hline Surgical complications & $2(1.6)$ & 124 & $3(20.0)$ & 15 & 0.041 \\
\hline Intraop durotomies & $5(4.0)$ & 124 & $1(6.7)$ & 15 & 0.440 \\
\hline Medical complications & $4(3.2)$ & 124 & 0 & 15 & 0.620 \\
\hline Readmit <30 days & $2(1.6)$ & 124 & 124 & 14 & 0.015 \\
\hline Mean follow-up length in yrs & $2.1 \pm 1.6$ & $4.2 \pm 2.7$ & & 15 \\
\hline * Level of significance: 0.00320. Values are presented as the number of patients (\%) unless stated otherwise. Mean values are expressed as \\
the mean \pm 1 SD. Values in boldface are statistically significant. & & & &
\end{tabular}

largest group of patients receiving endoscopic treatment for sagittal synostosis. Among 139 cases meeting the study criteria, the mean age was 3.6 months, perioperative blood transfusion rate was 9\%, mean EBL was $29 \mathrm{ml}$, mean operative time was 58 minutes, and mean length of hospital stay was 1.07 days. ${ }^{16}$ Jimenez and Barone also reported similar findings for endoscopic correction of coronal synostosis in 115 patients. ${ }^{14} \mathrm{We}$ included all types of craniosynostosis in our study population and found a mean age of 3.4 months, intraoperative blood transfusion rate of 5.0\%, mean EBL of $36.1 \mathrm{ml}$, mean operative time of 71.3 minutes, and mean length of hospital stay of 1.1 days for endoscopic patients. All of these measures were significantly decreased from those of similar patients receiving open surgery, with the important difference that the patients undergoing open procedures were older on average (15.5 months). Therefore, we conclude that although the improved metrics for endoscopic surgery reported by Barone et al. are reproducible across different patient populations, the advantages of minimally invasive surgery are partially confounded by selection bias for more advanced presentations, and potentially more difficult operations, in the open group. For comparison, Engel et al. reviewed 54 cases of open operations for nonsyndromic metopic synostosis with a median age at operation of 11.5 months (range 6-52 months) and found a median EBL of less than $255 \mathrm{ml}$ (range 80-600 $\mathrm{ml}), 100 \%$ transfusion rate, and average length of hospital stay of 5 days. ${ }^{8}$ These results are similar to our findings for nonsyndromic open operations (Table 2).

Chan et al. directly compared metrics in endoscopic versus open craniosynostosis surgery by examining a cohort of 21 patients who underwent open procedures and 36 who underwent endoscopic procedures receiving treatment for all types of craniosynostosis. ${ }^{5}$ They found a mean age at surgery of 10.56 months for open patients and 4.74 months for endoscopic patients. Our nonsyndromic patients had average ages of 15.5 months (open) and 3.4 months (endoscopic) at time of surgery. For their open cases, Chan et al. reported an average operating room time of 342 minutes, EBL of $280 \mathrm{ml}$, PRBC transfusion rate of $86 \%$, and length of hospital stay of 4.9 days. Their endoscopic patients had an average operating room time of 133 minutes, EBL of $74 \mathrm{ml}, \mathrm{PRBC}$ transfusion rate of $55 \%$, and length of stay of 1.25 days. Our results are largely comparable to those described in this previous study (Table 2). However, 2 differences between these 2 data sets should be noted. First, Chan et al. examined a combined cohort of syndromic and nonsyndromic synostosis patients, while we analyzed syndromic cases separately. Second, the prior study reported operating room times from when the patients entered the operating room until the moment they exited, instead of the actual surgical procedure lengths that we described. Taking these factors into account, our larger data set supports the notion set forth by previous studies that endoscopic synostosis surgery confers relative advantages over traditional open operations in the examined dimensions.

Our study sought to further elucidate the rates and natures of complications associated with endoscopic versus open craniosynostosis surgery, and to compare the results with the literature. Jimenez et al. reported no infections, air emboli, intraoperative durotomies, intraparenchymal injuries, postoperative hematomas, seizures, or intraoperative deaths in their endoscopic sagittal synostosis series. However, they did describe superficial skin irritation along the incisions in 5 patients..$^{16}$ In their endoscopic coronal synostosis series, ${ }^{14}$ Jimenez and Barone found no infections, 
sagittal sinus injury, postoperative hematomas, visual or ocular injuries, seizures, conversion to open procedures, or deaths. They did report 2 intraoperative durotomies, 2 minor scalp irritations, 3 calvarial defects, and 2 venous air emboli with a change in Doppler tones and decrease in end-tidal carbon dioxide, but no changes in blood pressure or oxygenation and of no clinical significance..$^{14}$ Gociman et al. presented a series of 46 patients with nonsyndromic sagittal synostosis who received endoscopic treatment and experienced no conversions to open approaches, reoperations, air emboli, cerebral parenchymal injuries, postoperative infections, hematomas, coagulopathies, CSF leaks, seizures, or deaths. They reported 2 intraoperative durotomies that were repaired, and 8 patients who displayed pyrexia. ${ }^{12}$ Finally, a recent study from our group found similar rates of delayed synostosis of uninvolved sutures between open and endoscopic procedures in nonsyndromic patients. ${ }^{34}$

In the present series, we classified events as surgical complications, intraoperative durotomies, or medical complications (Table 1). Within both our nonsyndromic and syndromic groups, we found comparable rates of intraoperative durotomies and surgical and medical complications between endoscopic and open approaches (Tables 2 and 3). Therefore, our conclusion is that we can advocate either technique in most situations regardless of syndrome status.

With respect to the literature for complications related to endoscopic procedures, we report comparable rates of events in both our nonsyndromic and syndromic endoscopic groups. Our rates in the nonsyndromic endoscopic group are perhaps elevated compared with prior studies because we were more inclusive in what we considered complications (Table 1) and because our large study population revealed rare events that are less likely to be observed in smaller series. Finally, although our series shows similar rates of complications between endoscopic and open synostosis operations, the ultimate decision regarding the most appropriate approach depends on a combination of complications, outcomes, and patient factors. To this end, we have previously found outcomes of the two approaches to be similar for correction of sagittal synostosis. ${ }^{28}$

Wood et al. presented a discussion of the merits of scalpel versus unipolar electrocautery skin incisions for craniosynostosis surgery and found no statistically significant difference in wound complications, which was in agreement with most human clinical and animal studies. ${ }^{33}$ Additionally, they suggested that, due to reported advantages of electrocautery including decreased incision time, improved hemostasis, and decreased postoperative pain, electrocautery is perhaps a more favorable method. However, they also acknowledged that the final decision to use scalpel or electrocautery for incisions is largely dependent on physician preference..$^{33}$ Although we did not have data for all subjects, we reported our experience with the use of scalpel versus electrocautery for initial skin incisions in this series. In general, we were much more likely to use scalpel for endoscopic procedures, while our open procedures were split evenly between the two methods (Table 2). To date, we have observed no notable differences in cosmetics or complications related to our decisions to use scalpel versus electrocautery for skin incisions.
Previous studies have provided strong evidence that TXA administration reduces blood loss..$^{13}$ We attempted to corroborate these findings with our patients. First, looking at all nonsyndromic patients (endoscopic and open), we found that patients who received TXA $(\mathrm{n}=30)$ had higher EBL $(246.7 \pm 225.4 \mathrm{ml})$ than those who did not receive TXA $(137.5 \pm 168.2 \mathrm{ml}, \mathrm{n}=215)$. Because this result is contrary to previous findings, we conducted subgroup analyses to determine the cause of the discrepancy. Only 8 endoscopic patients were documented to have received TXA, so we did not have enough statistical power to draw conclusions based on this group. However, looking at only open nonsyndromic cases with available data, and separating by plastic surgeons, we found that K.B.P. used TXA in all of his 19 open cases, whereas A.S.W. used it in only 3 of his 62, and A.A.K. did not administer TXA to any of his open nonsyndromic patients. Therefore, the previous results are driven almost exclusively by K.B.P.'s open cases in the TXA group versus all 3 surgeons' in the non-TXA group. Any findings are likely a result of more general variations between the surgeons rather than TXA administration. Based on these analyses, we conclude that our data lack statistical power and systematic design needed to corroborate findings from previous studies. Additionally, they reflect clear variations in practices between surgeons and suggest a need for a standardized protocol addressing TXA administration for future investigation.

One potential drawback of our study is that our standard follow-up for patients receiving endoscopic operations is shorter than those receiving open surgery. Therefore, we are presenting earlier outcomes of endoscopic surgery. Longer follow-ups for both endoscopic and open synostosis corrections are needed to better understand and compare outcomes. Another note is that because the endoscopic procedures are limited to children younger than 6 months, open techniques are still the default for older patients. Older children are likely to bleed more, have thicker bone, and need more operative time, all of which might be reflected in our comparisons. We attempted to control for age by performing a subgroup analysis of nonsyndromic patients younger than 150 days at the time of surgery (Table 6), but still had significantly different mean ages between endoscopic and open groups due to small sample sizes. Nevertheless, this analysis yielded results similar to those obtained from the full series.

We used EBL as a point of comparison between open and endoscopic approaches in our study, but it should be acknowledged that this is a somewhat subjective estimation based on suction canister contents, sponges, and irrigation amounts with potential for variation between surgeons and anesthesiologists. Additionally, EBL is directly related to the weight and EBV of the child, which we attempted to address with our calculation of the EBL/EBV ratio.

Finally, the decision of whether and when to perform transfusions in patients of varying ages is subject to a number of nuances that may have affected our results but not been explicitly addressed. For example, transfusion decisions in younger children are potentially more complicated due to compounded effects of preoperative fasting status, volume loading, and physiological nadir leading to different thresholds for giving blood in younger versus 
older patients. Additionally, the final decision to perform transfusions is highly subjective and varies not just with surgeons, but also ICU staff and anesthesiologists. Combined with the difference in mean ages between open and endoscopic cases, these factors all potentially contribute to the results of our transfusion rate comparisons.

\section{Conclusions}

We present the largest direct comparison to date between endoscopic and open interventions for all types of craniosynostosis. Compared with open operations, endoscopic procedures were associated with decreased EBL, transfusions, procedure lengths, and lengths of hospital stay. Rates of intraoperative durotomies, surgical, and medical complications were comparable between endoscopic and open techniques. These results are in agreement with previous series, in that although open reconstruction remains the only option in older children and results tend to be excellent, endoscopic techniques seem to offer clear advantages in appropriate populations such as decreased blood loss, shorter surgical times, and shorter hospitalizations without an increase in morbidity.

\section{References}

1. Barone CM, Jimenez DF: Endoscopic craniectomy for early correction of craniosynostosis. Plast Reconstr Surg 104:1965-1975, 1999

2. Barone CM, Jimenez DF: Endoscopic approach to coronal craniosynostosis. Clin Plast Surg 31:415-422, vi, 2004

3. Bergmans B, Kammeraad JAE, van Adrichem LNA, Staals LM: Craniosynostosis surgery in an infant with a complex cyanotic cardiac defect. Paediatr Anaesth 24:788-790, 2014

4. Berry-Candelario J, Ridgway EB, Grondin RT, Rogers GF, Proctor MR: Endoscope-assisted strip craniectomy and postoperative helmet therapy for treatment of craniosynostosis. Neurosurg Focus 31(2):E5, 2011

5. Chan JWH, Stewart CL, Stalder MW, St Hilaire H, McBride L, Moses MH: Endoscope-assisted versus open repair of craniosynostosis: a comparison of perioperative cost and risk. $\mathbf{J}$ Craniofac Surg 24:170-174, 2013

6. Doumit GD, Papay FA, Moores N, Zins JE: Management of sagittal synostosis: a solution to equipoise. J Craniofac Surg 25:1260-1265, 2014

7. Eley KA, Johnson D, Wilkie AOM, Jayamohan J, Richards P, Wall SA: Raised intracranial pressure is frequent in untreated nonsyndromic unicoronal synostosis and does not correlate with severity of phenotypic features. Plast Reconstr Surg 130:690e-697e, 2012

8. Engel M, Thiele OC, Mühling J, Hoffmann J, Freier K, Castrillon-Oberndorfer G, et al: Trigonocephaly: results after surgical correction of nonsyndromatic isolated metopic suture synostosis in 54 cases. J Craniomaxillofac Surg 40:347-353, 2012

9. Erşahin Y: Endoscope-assisted repair of metopic synostosis. Childs Nerv Syst 29:2195-2199, 2013

10. Fearon JA: Evidence-based medicine: Craniosynostosis. Plast Reconstr Surg 133:1261-1275, 2014

11. Fisher DC, Kornrumpf BP, Couture D, Glazier SS, Argenta LC, David LR: Increased incidence of metopic suture abnormalities in children with positional plagiocephaly. J Craniofac Surg 22:89-95, 2011

12. Gociman B, Marengo J, Ying J, Kestle JRW, Siddiqi F: Minimally invasive strip craniectomy for sagittal synostosis. J Craniofac Surg 23:825-828, 2012
13. Goobie SM, Meier PM, Pereira LM, McGowan FX, Prescilla RP, Scharp LA, et al: Efficacy of tranexamic acid in pediatric craniosynostosis surgery: a double-blind, placebo-controlled trial. Anesthesiology 114:862-871, 2011

14. Jimenez DF, Barone CM: Early treatment of coronal synostosis with endoscopy-assisted craniectomy and postoperative cranial orthosis therapy: 16-year experience. J Neurosurg Pediatr 12:207-219, 2013

15. Jimenez DF, Barone CM: Endoscopic technique for sagittal synostosis. Childs Nerv Syst 28:1333-1339, 2012

16. Jimenez DF, Barone CM, McGee ME, Cartwright CC, Baker CL: Endoscopy-assisted wide-vertex craniectomy, barrel stave osteotomies, and postoperative helmet molding therapy in the management of sagittal suture craniosynostosis. J Neurosurg 100 (5 Suppl Pediatrics):407-417, 2004

17. Kirman CN, Tran B, Sanger C, Railean S, Glazier SS, David LR: Difficulties of delayed treatment of craniosynostosis in a patient with Crouzon, increased intracranial pressure, and papilledema. J Craniofac Surg 22:1409-1412, 2011

18. Lee HQ, Hutson JM, Wray AC, Lo PA, Chong DK, Holmes $\mathrm{AD}$, et al: Analysis of morbidity and mortality in surgical management of craniosynostosis. J Craniofac Surg 23:1256-1261, 2012

19. Lee HQ, Hutson JM, Wray AC, Lo PA, Chong DK, Holmes $\mathrm{AD}$, et al: Changing epidemiology of nonsyndromic craniosynostosis and revisiting the risk factors. J Craniofac Surg 23:1245-1251, 2012

20. Meier PM, Goobie SM, DiNardo JA, Proctor MR, Zurakowski D, Soriano SG: Endoscopic strip craniectomy in early infancy: the initial five years of anesthesia experience. Anesth Analg 112:407-414, 2011

21. Meier PM, Guzman R, Erb TO: Endoscopic pediatric neurosurgery: implications for anesthesia. Paediatr Anaesth 24:668-677, 2014

22. Nguyen DC, Patel KB, Skolnick GB, Naidoo SD, Huang AH, Smyth MD, et al: Are endoscopic and open treatments of metopic synostosis equivalent in treating trigonocephaly and hypotelorism? J Craniofac Surg 26:129-134, 2015

23. Pagnoni M, Fadda MT, Spalice A, Amodeo G, Ursitti F, Mitro V, et al: Surgical timing of craniosynostosis: what to do and when. J Craniomaxillofac Surg 42:513-519, 2014

24. Patel A, Terner J, Travieso R, Clune JE, Steinbacher D, Persing JA: On Bernard Sarnat's 100th birthday: pathology and management of craniosynostosis. J Craniofac Surg 23:105112,2012

25. Ridgway EB, Berry-Candelario J, Grondin RT, Rogers GF, Proctor MR: The management of sagittal synostosis using endoscopic suturectomy and postoperative helmet therapy. J Neurosurg Pediatr 7:620-626, 2011

26. Sanger C, David L, Argenta L: Latest trends in minimally invasive synostosis surgery: a review. Curr Opin Otolaryngol Head Neck Surg 22:316-321, 2014

27. Schaller BJ, Filis A, Merten HA, Buchfelder M: Premature craniosynostosis - the role of skull base surgery in its correction. A surgical and radiological experience of 172 operated infants/children. J Craniomaxillofac Surg 40:195-200, 2012

28. Shah MN, Kane AA, Petersen JD, Woo AS, Naidoo SD, Smyth MD: Endoscopically assisted versus open repair of sagittal craniosynostosis: the St. Louis Children's Hospital experience. J Neurosurg Pediatr 8:165-170, 2011

29. Singer S, Bower C, Southall P, Goldblatt J: Craniosynostosis in Western Australia, 1980-1994: a population-based study. Am J Med Genet 83:382-387, 1999

30. Teichgraeber JF, Baumgartner JE, Viviano SL, Gateno J, Xia JJ: Microscopic versus open approach to craniosynostosis: a long-term outcomes comparison. J Craniofac Surg 25:1245-1248, 2014

31. Vogel TW, Woo AS, Kane AA, Patel KB, Naidoo SD, Smyth 
MD: A comparison of costs associated with endoscope-assisted craniectomy versus open cranial vault repair for infants with sagittal synostosis. J Neurosurg Pediatr 13:324-331, 2014

32. Wong RK, Emelin JK, Meltzer HS, Levy ML, Cohen SR: Nonsyndromic craniosynostosis: the Rady Children's Hospital approach. J Craniofac Surg 23 (7 Suppl 1):2061-2065, 2012

33. Wood JS, Kittinger BJ, Perry VL, Adenola A, van Aalst JA: Craniosynostosis incision: scalpel or cautery? J Craniofac Surg 25:1256-1259, 2014

34. Yarbrough CK, Smyth MD, Holekamp TF, Ranalli NJ, Huang $\mathrm{AH}$, Patel KB, et al: Delayed synostoses of uninvolved sutures after surgical treatment of nonsyndromic craniosynostosis. J Craniofac Surg 25:119-123, 2014

\section{Disclosures}

Dr. Patel reports that he is a consultant with Stryker CMF and has a financial relationship with Hanger Clinic.

\section{Author Contributions}

Conception and design: Han, Nguyen, Skolnick, Yarbrough, Naidoo, Patel, Woo, Smyth. Acquisition of data: Han, Bruck. Analysis and interpretation of data: Han, Nguyen, Skolnick. Drafting the article: Han, Yarbrough. Critically revising the article: Han, Nguyen, Yarbrough, Patel, Kane, Woo, Smyth. Reviewed submitted version of manuscript: all authors. Statistical analysis: Nguyen, Skolnick. Administrative/technical/material support: Naidoo. Study supervision: Patel, Kane, Woo, Smyth.

\section{Supplemental Information}

Previous Presentations

Portions of this work were presented in poster form at the AANS/ CNS Section on Pediatric Neurological Surgery Annual Meeting, Amelia Island, FL, December 2-5, 2014.

\section{Correspondence}

Rowland H. Han, Department of Neurosurgery, Washington University School of Medicine, One Children's Pl., Ste. 4s20, St. Louis, MO 63110.email: hanr@wusm.wustl.edu. 